\title{
Autismo, Teoria da Mente e o Papel da Cegueira Mental na Compreensão de Transtornos Psiquiátricos
}

\author{
Autism, Theory of Mind and the Role of Mindblindness in the Understanding \\ of Psychiatric Disorders
}

\author{
Hélio Tonelli* \\ Instituto de Psiquiatria do Paraná, Curitiba, Brasil
}

\begin{abstract}
Resumo
Indivíduos portadores de transtornos do espectro do autismo podem ser acometidos por anormalidades qualitativas nos contatos sociais e padrões de comunicação. Muitos estudos demonstraram que uma grande quantidade destes indivíduos pode ter anormalidades no processamento cognitivo "Teoria da mente" (ToM), isto é, eles podem ter uma incapacidade de inferir os seus estados mentais e os de terceiros. Tal condição foi chamada de "cegueira mental" por Baron-Cohen (1995). Alguns autores recentemente aventaram a possibilidade de que indivíduos sofrendo de esquizofrenia e transtorno bipolar, da mesma forma que autistas, exibam cegueira mental, causando comprometimento em seu funcionamento social. Este artigo discute o conceito de cegueira mental e a visão de que se trata de uma habilidade exclusivamente humana. Em seguida, apresenta alguns testes disponíveis para avaliação da cegueira mental e, finalmente, mostra algumas evidências científicas de que déficits ToM podem afetar pacientes com outras condições psiquiátricas. Palavras-chave: Autismo; Cognição; Percepção social; Esquizofrenia; Transtorno bipolar.
\end{abstract}

\begin{abstract}
Individuals with autism spectrum disorders may suffer from qualitative abnormalities in social contacts and communication patterns. Many studies have shown that a great amount of those individuals might have abnormalities in the Theory of Mind (ToM) cognitive processing, i.e., they may lack the capability of inferring their and others' mental states. This condition was named "mindblindness" by Baron-Cohen (1995). Some authors have recently focused on the possibility that individuals who suffer from schizophrenia and bipolar disorder, like autistic people, show mindblindness, causing them to develop social impairments. This paper discusses the concept of mindblindness and the view that deems it as an exclusively human ability. Afterwards, it presents some of the available tests to evaluate mindblindness and, finally, shows some scientific evidences that ToM deficits might affect patients with other psychiatric conditions.

Keywords: Autism; Cognition; Social perception; Schizophrenia; Bipolar disorder.
\end{abstract}

O termo "autismo" é usado para descrever um transtorno global do desenvolvimento caracterizado por anormalidades qualitativas nas interações sociais e nos padrões de comunicação, bem como por um repertório restrito, estereotipado ou repetitivo de interesses e atividades (Dover \& Le Couteur, 2007). O conceito de "espectro do autismo" (EA) abrange indivíduos portadores de tais anormalidades de forma mais ou menos grave e inclui o Transtorno Autista propriamente dito como o transtorno prototípico, além do Transtorno de Asperger, do Transtorno de Rett, do Transtorno Desintegrativo da Infância e dos Transtornos globais do Desenvolvimento Sem Outra Especificação (American Psychiatric Association, 2002).

\footnotetext{
"Endereço para correspondência: Av. Cândido de Abreu, $n^{\circ}$ 526, cjto. 311-B, Centro Cívico, Curitiba, PR, Brasil, CEP 80530-905. E-mail: hatonelli@terra.com.br
}

Indivíduos portadores de transtornos do EA podem apresentar empobrecimento no processamento de emoções, no reconhecimento de faces, do controle do olhar, da capacidade de imitação, do uso de gestos, do uso da linguagem pragmática (metáfora e ironia) e do reconhecimento de pensamentos e sentimentos de si mesmos e de outras pessoas (Frith \& Happé, 1999). Em relação à dificuldade dos autistas em reconhecer seus próprios eventos mentais e os de terceiros, diz-se que estes pacientes carecem de uma "Teoria da mente", expressão utilizada para nomear a habilidade automática e espontânea de se atribuir estados mentais a si mesmo e a outras pessoas a fim de se poder predizer e explicar comportamentos, como será descrito adiante.

A expressão "Teoria da mente" (ToM) deriva de um prestigiado artigo publicado na década de setenta por um primatologista e um psicólogo, Premack e Woodruff, cujo título questionava se, a exemplo dos seres huma- 
nos, os chimpanzés também teriam uma "Teoria da mente" (1978).

Nosso discurso cotidiano é repleto de expressões que descrevem estados mentais e tentar explicar o comportamento e as interações entre seres humanos sem o uso destes descritores pode ser uma tarefa bastante difícil. Baron-Cohen (1995) mostrou que, frente à imagem de um indivíduo entrando em seu quarto, olhando ao redor do mesmo e depois saindo, explicações como "talvez ele quisesse encontrar algo que pensou ter deixado ali" ou "possivelmente ele tenha ouvido um barulho e tenha pensado que havia alguém ali" (as palavras em destaque são descritores de estados mentais) nos parecem mais apropriadas e naturais do que "possivelmente ele faça isso todo o dia, vá até seu quarto, dê uma olhada ao redor e depois saia" (sem descritores de estados mentais). BaronCohen (1995) cunhou o termo "cegueira mental" para descrever os déficits apresentados por indivíduos autistas no processamento ToM, isto é, na capacidade de inferir ou atribuir estados mentais a terceiros, comprometendo sua capacidade de interagir socialmente.

Recentemente, o interesse pelo estudo de possíveis prejuízos no processamento ToM em autistas se estendeu para outros transtornos mentais, como a esquizofrenia, o transtorno esquizotípico de personalidade e o transtorno bipolar, tanto no que tange à melhora da compreensão dos mecanismos subjacentes ao aparecimento destes quadros quanto à possibilidade de desenvolvimento de estratégias de prevenção e de tratamento das mesmas.

O presente trabalho tem como objetivo abordar os conceitos de "processamento ToM" e de "cegueira mental", as correlações destes com o neurodesenvolvimento saudável e patológico, as metodologias de estudo do processamento ToM e os resultados preliminares de pesquisas envolvendo portadores de outros transtornos mentais que não os transtornos do EA.

\section{Processamento ToM e Cegueira Mental}

O conceito de processamento ToM não se refere, de fato, a uma "teoria", mas a uma habilidade mental automática de se atribuir estados mentais a si mesmo e a outros indivíduos, com a finalidade principal de compreensão e predição de seus comportamentos. É importante destacar o papel da "automaticidade" desta habilidade, de maneira semelhante ao que ocorre, por exemplo, com os processos de decodifícação de estímulos sensoriais ambientais, nos quais também não ocorrem elaborações teóricas acerca do mundo, mas a disponibilização imediata, automática e espontânea de uma "versão" do mesmo que permita uma resposta comportamental adaptativa. Assim, ao abrirmos nossos olhos não elaboramos uma "Teoria Visual do Mundo", mas, simplesmente enxergamos o que há em nossa volta, automática e espontaneamente. Pelo fato da expressão "Teoria da mente" induzir a uma falsa interpretação do conceito, isto é, a de que essencialmente deveria ocorrer uma elaboração teórica subjacente ao processo de atribuição de estados mentais, alguns autores preferem o uso dos termos "mentalização" (que, a partir deste momento, empregarei como sinônimo de habilidades ToM) ou "adoção de uma postura intencional” (Dennett, 1987).

Dá-se o nome de ToM implícita (ToMi) à habilidade automática propriamente dita, de inferência de estados mentais, que permite um processamento rápido das informações oriundas do ambiente social; e de ToM explícita (ToMe) à capacidade de aprender as regras do jogo social, de forma a otimizar o convívio com outros seres humanos. ToMi e ToMe parecem recrutar circuitos neurais distintos e, do ponto de vista da eficiência, a ToMe é mais lenta, não permitindo um processamento online da informação social. Há mais de vinte anos Baron-Cohen, Leslie e Frith (1986) compararam a capacidade interpretativa de crianças normais, crianças portadoras de Síndrome de Down e de crianças autistas, de vinhetas ilustrando situações que exigiam integridade de habilidades ToM e vinhetas que não as exigiam. Os autores demonstraram que crianças autistas apresentavam prejuízo na compreensão das vinhetas que exigiam mentalização, bem como que sua performance era inferior tanto quando comparada com as das crianças normais quanto com as portadoras de Síndrome de Down. Atualmente, após muitos estudos investigando os déficits ToM de autistas, sabe-se que nem todos os indivíduos portadores do transtorno falham na interpretação de testes que exigem mentalização, mas que a maioria deles apresenta um atraso no desenvolvimento de habilidades ToM em relação a crianças normais. A razão para este atraso poderia ser explicada pelo prejuízo na configuração da ToMi, fazendo com que crianças autistas tenham de contar apenas com suas capacidades ToMe, as quais exigiriam mais tempo para seu completo desenvolvimento, uma vez que exigem aprendizado.

O comprometimento da mentalização em seres humanos foi também amplamente descrito em indivíduos portadores de esquizofrenia; nestes sujeitos, o estudo dos déficits ToM foi motivado pela existência de um distanciamento social inerente ao próprio transtorno, bem como pelo interesse em se explicar as razões pelas quais apresentavam sintomas psicóticos, como os delírios e as alucinações. Indivíduos esquizofrênicos, da mesma forma que autistas, parecem compensar as deficiências no processamento ToMi fazendo uso de circuitos cerebrais ToMe.

Pesquisas sugerindo indícios de "cegueira mental" em indivíduos portadores de autismo e esquizofrenia têm motivado a investigação desta condição em outros transtornos mentais. Achados positivos no que tange à ocorrência de problemas no processamento ToM em quaisquer transtornos mentais poderão auxiliar no desenvolvimento de estratégias preventivas e terapêuticas para os mesmos. 
Processamento ToM: Habilidade

Exclusivamente Humana?

A capacidade de mentalização parece ser exclusivamente humana, apesar de alguns psicólogos e primatologistas requererem que seja reconhecida em primatas antropóides. De fato, espécies sociais não-humanas são capazes de desempenhar comportamentos de sofisticação variável, os quais podem nos levar a acreditar que elas também possuem habilidades ToM, como caçar em grupos, criar proles, escolher parceiros, reconhecer outros indivíduos, reconhecer emoções básicas (como medo e raiva) em outros indivíduos, distinguir status social e fazer alianças. Contudo, estes animais podem fazer tudo isso sem mentalização, pois para serem realizados, tais comportamentos exigiriam apenas aprendizado de regras sociais e não representações mentais de estados mentais. Primatas não-humanos, apesar de toda a sua flagrante inteligência e capacidade imitativa, não parecem ter desenvolvido habilidades de mentalização, apesar de muitos de seus comportamentos os distinguirem de outras espécies e serem muito mais semelhantes a determinados comportamentos vistos unicamente na espécie humana. Por exemplo, ao contrário da maioria dos mamíferos, em determinadas situações, macacos podem contar com o auxílio de outros co-específicos, além de seus próprios recursos; assim como são sensíveis a características individuais e relações hierárquicas entre eles (Frith \& Frith, 1999). Outros "truques" destes animais incluem o uso de estratégias de engano cujo objetivo principal é o de manipular o comportamento de outros co-específicos. Tais ardis, todavia, não parecem derivar da capacidade de mentalização, mas sim de um rápido aprendizado a partir de vários reforçadores coincidentes (Frith \& Frith, 1999). Até o momento não foi possível demonstrar evidências inequívocas de habilidades ToM em primatas.

Estudos conduzidos acerca do desenvolvimento cognitivo em primatas antropóides e humanos durante as três últimas décadas têm dado suporte à chamada Hipótese da Encefalização, que se refere ao aumento progressivo no tamanho relativo do cérebro, particularmente do córtex, ao longo da evolução dos mamíferos, em particular, dos primatas (Donald, 1993). Várias formas de se compreender o desenvolvimento da mente humana e de suas peculiaridades à luz da Hipótese da Encefalização parecem coexistir. Correntes mais radicais sustentam que o aumento do poder de processamento cognitivo dos humanos decorre essencialmente do aumento do volume cortical. Outras atribuem a encefalização a pressões evolutivas, tais como a necessidade de mapear geograficamente o ambiente e de produzir ferramentas que auxiliem a caça, o abrigo e a autodefesa.

Interessado nas razões por detrás do peculiar comportamento social de primatas antropóides, Dunbar (2002) propôs que exista uma relação entre o que chamou de "razão de neocórtex" e o tamanho médio de grupos destes animais. Por "razão de neocórtex" deve-se entender a relação entre o volume do neocórtex e o volume do res- tante do cérebro. Tal equação de dimensão encefálica elimina a possibilidade de que diferenças dos volumes neocorticais medidos devam-se a diferenças atribuíveis apenas ao tamanho corporal. Portanto, indivíduos vivendo em grupos sociais maiores apresentariam maiores razões neocorticais. A tese de Dunbar é a de que o tamanho dos grupos sociais exerceu pressão evolutiva para crescimento do neocórtex nos primatas antropóides, em virtude do aumento da complexidade das relações sociais e das exigências em termos de processamento cerebral de informações relativas a estas relações, o que teria propiciado, em última análise, o desenvolvimento das avançadas aptidões humanas de atribuição de estados mentais.

Assim, graças ao desenvolvimento da mentalização, além de conseguirmos fazer tudo o que animais sociais fazem sem ToM, somos capazes de - utilizando de um poderoso processador cognitivo social - negociar, enganar, ensinar, demonstrar e reconhecer emoções complexas, além de manipular pensamentos, permitindo uma previsão comportamental baseada neste mecanismo de inferência de emoções e crenças com consideráveis probabilidades de acerto. Este processador constituir-se-ia, em síntese, de um mecanismo gerador de representações mentais acerca de estados mentais.

Representações mentais podem ser consideradas "cópias" do mundo, geradas a partir de circuitos cerebrais que computam a informação sobre o meio ambiente, conduzida pelos órgãos do sentido (Gazzaniga, Ivry, \& Mangun, 2006). Deve-se aqui salientar que a configuração de tais "cópias" envolve um importante caráter de subjetividade, já que cada indivíduo assimila suas representações mentais de maneira distinta. A geração destas "cópias" por circuitos cerebrais tem como principal finalidade o planejamento comportamental baseado na simulação do ambiente - uma vez que qualquer coisa pode ser, em princípio, representada mentalmente - com a vantagem deste planejamento ser realizado de forma eficiente e segura, isto é, sem a perda de tempo e os riscos de uma manipulação direta de variáveis do meio.

Representações mentais de estados mentais são modelos das mentes de terceiros (e da própria mente) e participam do planejamento dos comportamentos a serem adotados diante da interação com outros humanos. A maneira pela qual tais representações são geradas ainda é motivo de controvérsia entre autores, que se dividem, basicamente, em três grupos (Brüne \& Brüene-Cohrs, 2006; Flavell, 1999). O primeiro considera que a experiência individual é fundamental na configuração das habilidades ToM, que seriam computadas a partir do contínuo aprendizado adquirido através do convívio social. Tal concepção é denominada "Teoria - Teoria". O segundo grupo, chamado de "Teoria modular", não valoriza da mesma forma o papel da experiência no desenvolvimento da mentalização, mas propõe a existência de "módulos" cerebrais configurados de maneira inata para a atribuição de estados mentais. O último grupo postula que o processamento ToM se desenvolve a partir de uma capaci- 
dade de simulação dos estados mentais de terceiros, possível graças à apreciação dos próprios estados mentais. Por isso, sua hipótese é denominada "Teoria da simulação".

\section{Desenvolvimento dos Circuitos Neurais Relacionados ao Processamento ToM}

Para Alan Leslie (1994), um teórico modular, as representações de estados mentais geradas pelo processador ToM poderiam ser incluídas em três categorias distintas: representações de estados mentais volitivos, perceptuais e epistêmicos, que serão detalhados a seguir.

Comportamentos ou ações de terceiros estão entre os mais importantes estímulos para os humanos e, embora possam conduzir informações não enviadas voluntariamente, somos capazes de compreender o que os outros estão fazendo e porque estão fazendo.

Esta capacidade de entender ações alheias configura-se não através de uma simples leitura de movimentos corporais, mas de ações intencionais, a partir da atribuição a terceiros de atitudes proposicionais, isto é, crenças, desejos, pensamentos e intenções, dirigindo o comportamento e tornando-o previsível (Rizzolatti \& Sinigaglia, 2007), o que, em última análise, caracteriza o processo de mentalização.

No final do século XIX o filósofo germano-austríaco Franz Brentano propôs que a marca registrada da mente seria sua propriedade de intencionalidade, isto é, a possibilidade que um indivíduo tem de apontar ou dirigir-se a outras coisas que não a si mesmo (Gómez, 2009). De fato, um ato mental compreende invariavelmente um "alvo" (pensar em alguma coisa, desejar algo) (Gómez, 2009). O conceito de intencionalidade de Brentano salienta, portanto, o caráter da ação de um agente como baseado em um objetivo e este conceito pode nos auxiliar na compreensão das configurações do processador cognitivo social, que poderia ser concebido inicialmente como um sistema "detector de intencionalidade", envolvido, a priori, na detecção de qualquer ação por parte de um agente ambiental. Tal ação poderia ser puramente física e materializada através de um comportamento motor, ou essencialmente subjetiva ou virtual, expressa, por exemplo, através de um pensamento (Rochat, 2007).

Do ponto de vista da psicologia evolucionária, a capacidade de deteç̧ão de intencionalidade (e, por conseguinte, a de mentalização) não se desenvolveu preliminarmente para desempenhar especificamente esta tarefa e pode ser compreendida como a resultante de um novo emprego de habilidades cognitivas pré-existentes. Isto é, a evolução pode ter exercido pressão para que o cérebro ancestral recrutasse sistemas neurais originalmente envolvidos com outras funções e os utilizasse como substrato para os futuros circuitos de processamento da intencionalidade. As funções cerebrais a que são mais comumente atribuídas as origens da habilidade de detecção de intencionalidade são a percepção visual de movimento, a capacidade de atenção visual compartilhada e a habilidade de representar ações objetivodirigidas (Frith \& Frith, 1999).

A percepção visual de movimento é uma importante fonte de informação sensorial, particularmente para humanos e primatas antropóides, que são capazes de, sem dificuldade, distinguir entre dois tipos distintos de movimento: biológico (executado por seres vivos) e não biológico. Esta distinção permite um aumento na precisão da detecção de presas e potenciais predadores, além de oportunidades de acasalamento (Blakemore \& Decety, 2001). Experimentos em que luzes são acopladas às principais articulações de humanos, cujos movimentos são gravados em um ambiente escuro, demonstraram que não apenas humanos adultos, mas bebês de apenas 3 meses de idade, estão aptos a discriminar movimentação biológica de não biológica (Blakemore \& Decety, 2001). Outras habilidades que ancestralmente não processavam especificamente a detecção de intencionalidade incluem o peculiar interesse de bebês por objetos que se movem por autopropulsão e por rostos, expressões faciais e, particularmente, os olhos e a direção do olhar de terceiros (Flavell, 1999), a qual já pode ser seguida por crianças também muito pequenas. Tal habilidade é denominada atenção visual compartilhada e parece ser fundamental para a configuração de capacidades cognitivas sociais saudáveis em um contexto de experiência diádica.

Portanto, a partir de processos essencialmente visuais - percepção acurada e maior interesse por movimento biológico e pela orientação do olhar de outro humano (atenção compartilhada) - configura-se a capacidade mental de perceber como as pessoas e outros seres animados se relacionam subjetivamente a objetos, isto é, esboça-se a habilidade de construir representações mentais objetivo-dirigidas.

Baron-Cohen (1995) afirma que, além dos mecanismos de detecção de movimentação autopropulsionada ou biológica e atenção visual compartilhada, mais um sistema neural seria necessário para o pleno desenvolvimento de adequada habilidade de mentalização. Este sistema foi denominado "Mecanismo Teoria da mente" ou ToMM por Alan Leslie (1994) e se correlaciona especificamente à inferência de estados mentais a partir de comportamentos.

Desta forma, os circuitos cerebrais detectores de movimentos autopropulsionados ou biológicos processam a leitura de comportamentos a partir de estados mentais volitivos (desejo e objetivo), a atenção compartilhada o faz através de estados mentais perceptuais e o módulo ToMM, por meio da criação de representações de estados mentais epistêmicos (fingir, pensar, saber, imaginar, acreditar, etc.). Além de gerar deste tipo de representação, o módulo ToMM permite a integração das tarefas dos três módulos em questão (Baron-Cohen, 1995).

Da mesma forma que as representações de estados volitivos e perceptuais, as representações epistêmicas são geradas a partir do rastreamento do agente. A distinção 
entre o rastreamento puramente perceptual e motor e o rastreamento epistêmico está na forma como eles são realizados: no primeiro caso, o mesmo se dá pelo acompanhamento direto do agente através do emprego de um sistema sensório-motor; no segundo, em virtude do agente não poder ser rastreado por métodos puramente perceptivos, faz-se necessário o uso de informações indiretas a respeito do mesmo, como a reflexão e a memória (Bullot \& Rysiew, 2007).

\section{Tarefas ToM}

Diversos testes estão disponíveis e já foram utilizados em estudos comparando a performance ToM de crianças e adultos, saudáveis e portadores de transtornos psiquiátricos ou do desenvolvimento. De maneira geral, consistem de tarefas verbais ou não verbais (ou compostas) que, para serem adequadamente executadas, exigem habilidade ToM intacta. As tarefas ToM devem ser administradas conjuntamente com tarefas-controle (que não exigem mentalização), a fim de se eliminar a possibilidade de baixas pontuações nas tarefas ToM decorrerem de dificuldades de compreensão geral dos testes aplicados.

Wimmer e Perner (1983) propuseram o Sally - Anne Task (SAT), que se popularizou inicialmente entre os pesquisadores por sua simplicidade em avaliar a capacidade de detecção de uma falsa crença. O cenário descrito pelo SAT consiste em uma pequena vinheta envolvendo duas personagens, Sally e Anne (estas personagens podem ser apresentadas através de desenhos, bonecos ou encenadas por atores). A vinheta é apresentada a seguir:

Sally possui uma bola e uma cesta e Anne possui uma caixa. Sally coloca sua bola dentro de sua cesta e sai de cena. Enquanto está fora, Anne pega a bola dentro da cesta de Sally e a coloca em sua caixa. Sally volta. É, então, perguntado ao examinado onde ele acha que Sally irá procurar por sua bola: em seu cesto ou na caixa de Anne? A situação ilustra de maneira simples a falsa crença sustentada por Sally, ou seja, a de que a sua bola se encontra ainda dentro de sua cesta, pois ela não viu Anne trocar o brinquedo de lugar. A maior parte das crianças com mais de quatro anos de idade e indivíduos sem problemas no processamento ToM responderão que Sally deverá procurar por sua bola no cesto. Crianças menores de quatro anos e indivíduos com problemas no processamento ToM não conseguirão representar mentalmente de forma adequada a situação mental de Sally e tenderão a responder que ela deverá procurar por sua bola na caixa de Anne, na medida em que terão problemas para representar o estado mental de Sally, a qual não sabe que sua bola foi trocada de lugar durante o tempo em que esteve ausente.

Outro teste visual bastante popular para avaliação de uma falsa crença é o Smarties Test (ST) (Hogrefe, Wimmer, \& Perner, 1986), que apresenta a situação em que uma embalagem muito conhecida de doces (e que dá nome à tarefa) é mostrada a um personagem, ao qual é perguntado sobre seu conteúdo. O mesmo responde imediatamente: "doces". Em seguida, a embalagem é aberta e é mostrado ao personagem que, na realidade, ela contém vários lápis, ao invés de doces. Pergunta-se ao examinado, então, o que ele acha que outra criança (para a qual nada foi dito a respeito do conteúdo da embalagem) responderá sobre o que há naquela caixa de doces.

O John and Mary Test ([JMT], Perner \& Wimmer, 1985), por sua vez, avalia a compreensão de falsas crenças de segunda ordem, isto é, falsas crenças que alguém pode ter em relação às crenças de outra pessoa. Por exemplo, "João acredita que Maria pensa que neva em Salvador" será uma falsa crença de segunda ordem se Maria de fato não acreditar que neva em Salvador. O JMT compreende uma história em que os personagens John e Mary são informados independentemente sobre a mudança de lugar em que deverá haver um sorveteiro. Tanto John quanto Mary sabem desta mudança de lugar, mas John não sabe que Mary também sabe de tal mudança. John, portanto, sustenta uma falsa crença de segunda ordem acerca da crença de Mary, isto é, ele pensa que Mary ainda acredita que o sorveteiro está no antigo lugar. A compreensão da falsa crença de segunda ordem é acessada através da pergunta "Onde John acha que Mary vai se dirigir para comprar o sorvete?".

Alguns autores têm utilizado vinhetas compostas por cartoons ou desenhos de cenários ToM mais complexos - envolvendo cooperação, sabotagem e traição entre os protagonistas - na avaliação do processamento ToM (Brüne, 2003; Brüne \& Bodenstein, 2005; Corcoran, Cahil, \& Frith, 1997) de suas amostras, associando-as ou não a tarefas ToM verbais, como o Hinting Task ([HT], Corcoran, Mercer, \& Frith, 1995), o Eyes Test ([ET], Baron-Cohen, Wheelwright, Hill, Raste, \& Plumb, 2001) e o Faux Pas Test ([FPT], Stone, Baron-Cohen, \& Knight, 1998).

O HT é composto por dez vinhetas apresentadas na forma de texto, descrevendo a interação entre dois personagens, juntamente de um extrato de seu diálogo, onde um deles faz uma sugestão ao outro que, para ser compreendida, exige inferência de estados mentais. O FPT, da mesma forma que o HT, consiste de uma tarefa puramente verbal, composta por 10 histórias em que um dos personagens diz alguma coisa que não deveria ter dito, cometendo um faux pas (ou, em linguagem corriqueira, uma "gafe"). A história deve ser lida em voz alta ao examinado, que deverá identificar se algum dos personagens disse algo que não deveria ter dito e qual deles o fez.

O ET é uma tarefa composta (verbal e não verbal) baseada na avaliação da capacidade de descrição de estados mentais a partir da observação de fotografias das regiões em torno dos olhos de pessoas desconhecidas. A descrição de estado mental é feita através da melhor opção de descritor dentre quatro possibilidades apresentadas por fotografia.

Alguns pesquisadores examinaram, ainda, a relação entre a integridade do processamento ToM e a capaci- 
dade de compreender elementos de linguagem pragmática como a metáfora e a ironia (Langdon \& Coltheart, 2004).

De maneira geral, todas as tarefas até o momento empregadas na avaliação da capacidade de inferência de estados mentais, tanto em adultos quanto em crianças, saudáveis ou portadores de transtornos mentais ou do desenvolvimento, podem ser criticadas seja por terem sido desenvolvidas tendo em vista o estudo de um determinado transtorno em particular, seja por serem puramente verbais ou puramente não verbais; ou, ainda, por não permitirem uma avaliação precisa do processo de inferência de estados mentais de forma semelhante ao que ocorre na realidade. Isto é, por permitirem, de certa forma, algum tipo de reflexão, estas tarefas não simulariam o processamento ToM online - automático e espontâneo - que é esperado que aconteça nos indivíduos saudáveis, permitindo assim, ao menos parcialmente, recrutar circuitos ToMe, baseados em aprendizado de regras sociais. Visando contornar este problema, têm sido desenvolvidas tarefas compostas por filmes gravados contendo interações entre pessoas (Mc Donald, Flanagan, Rollins, \& Kinch, 2003). Estas tarefas visam mimetizar melhor as condições naturais em que o processamento da mentalização ocorre.

Muitas das críticas dirigidas aos trabalhos envolvendo a investigação do processamento ToM através do emprego das tarefas supradescritas dizem respeito à não utilização de tarefas cognitivas gerais - principalmente função executiva, pela utilização de medidas de atenção, controle inibitório, flexibilidade cognitiva e velocidade de processamento - e QI, pelo fato de que tarefas ToM também recrutam circuitos não relacionados especificamente ao processamento ToM para serem adequadamente realizados. Muitos estudos bem conduzidos têm, contudo, demonstrado situações em que ocorrem prejuízos na performance em tarefas ToM, a despeito de escores normais em tarefas cognitivas gerais ou QI.

\section{Cegueira Mental e Transtornos Psiquiátricos}

Resultados de trabalhos sugerindo a existência de problemas no processamento ToM em indivíduos portadores de autismo estimularam a investigação a respeito da existência de cegueira mental em outros transtornos psiquiátricos como a esquizofrenia e o transtorno bipolar. Além destes transtornos, existem muitos dados a respeito de déficits de mentalização em indivíduos vulneráveis à esquizofrenia, como os portadores de transtorno esquizotípico de personalidade e os parentes em primeiro grau de esquizofrênicos.

Chris Frith (1992) sugeriu pela primeira vez que os sintomas da esquizofrenia poderiam ser decorrentes de prejuízos em habilidades ToM nestes indivíduos. Frith sugeriu que os pacientes com predomínio de sintomas das dimensões negativa e desorganizada (ligadas, respectivamente, à presença de apatia, falta de iniciativa $\mathrm{e}$ alogia; e pensamento e comportamentos grosseiramente desorganizados, em oposição à sintomatologia positiva que caracteriza os delírios e alucinações dos indivíduos esquizofrênicos) teriam comprometimento da capacidade de representar os estados mentais dos outros e de si mesmos, enquanto os pacientes paranóides apresentariam apenas comprometimento da capacidade de representar os estados mentais dos outros. Logo em seguida, Hardy-Baylé, Passerieux, Claudel, Olivier e Chevalier (1994) argumentaram que o prejuízo das habilidades ToM na esquizofrenia apenas refletiria o déficit executivo destes pacientes, de forma que alterações nas habilidades ToM seriam proporcionais à gravidade da síndrome disexecutiva. Para estes autores, pacientes paranóides sem sintomas da dimensão desorganizada não teriam déficits de habilidades ToM.

Muitos estudos propuseram-se a correlacionar as alterações das habilidades ToM em esquizofrênicos e os sintomas da esquizofrenia, testando os modelos propostos por Frith e Hardy-Baylé. Brüne (2005), revisando o assunto, concluiu que os dados disponíveis parecem confirmar a hipótese de que os pacientes com predomínio de sintomas negativos e de desorganização têm piores pontuações na testagem de habilidades ToM do que pacientes com predomínio de sintomas positivos. O modelo de Hardy-Baylé parece confirmar-se apenas parcialmente, já que muitos estudos não sustentam sua hipótese de que pacientes paranóides sem extensa sintomatologia desorganizada não apresentariam prejuízos de habilidades ToM. Pelo fato de que o processamento ToM deve funcionar em profunda consonância com as habilidades cognitivas gerais, é que o estudo dos prejuízos de habilidades ToM em indivíduos esquizofrênicos deve controlar as variáveis cognitivas "não-ToM" que possam interferir nos resultados. Déficits atencionais, executivos e baixos escores de inteligência objetivamente influenciam a mensuração das habilidades ToM, mas a comparação de indivíduos normais com esquizofrênicos no que tange à performance em testes que avaliam estas habilidades continua favorecendo significativamente controles saudáveis quando são controlados os fatores relacionados à cognição.

Duas metanálises publicadas recentemente abordaram o assunto (Bora, Yucel, \& Pantelis, 2009; Sprong, Schothorst, Vos, Hox, \& van Engeland, 2007). Sprong et al. avaliaram 29 estudos cujos resultados sugeriram que o desempenho de pacientes esquizofrênicos nas tarefas empregadas para mensuração de habilidades ToM foi significativamente inferior que a de controles saudáveis. Além disso, variáveis como QI, gênero e idade não afetaram significativamente este resultado, apesar de outras importantes variáveis, como uso de medicação e duração e gravidade da doença não poderem ter sido examinadas por falta de informação em muitos dos estudos incluídos. Bora et al. (2009), por sua vez, avaliaram 36 estudos, concluindo que indivíduos em esquizofrenia podem apresentar comprometimento na execução 
de tarefas ToM em relação a indivíduos saudáveis mesmo em fases de remissão da psicose, embora tal comprometimento seja menos acentuado. Apesar de sugerirem que déficits intelectuais dos indivíduos esquizofrênicos possam estar por trás deste comprometimento nas fases de remissão, os autores não descartam a importância de que novos estudos continuem examinando o caráter de traço-dependência da cegueira para estados mentais nestas populações.

A possibilidade de que problemas no processamento da inferência de estados mentais tenham um caráter traço-dependente - ou seja, eles existiriam, embora de forma atenuada, até mesmo nos períodos de remissão dos sintomas psicóticos da esquizofrenia - tem estimulado a procura por estes déficits em populações de indivíduos vulneráveis para este transtorno, como os parentes em primeiro grau de indivíduos esquizofrênicos e portadores de transtorno esquizotípico de personalidade (TEP). Uma importante vantagem do estudo destas populações reside no fato de que indivíduos vulneráveis via de regra não fazem uso de medicação antipsicótica, um dos fatores potencialmente comprometedores do desempenho cognitivo em indivíduos esquizofrênicos.

Recentemente Tonelli, Alvarez e da Silva (2009) revisaram 15 estudos abordando as relações entre diferentes formas de vulnerabilidade à psicose e alterações para mais ou para menos no processamento ToM. Os estudos avaliados abrangiam não somente o emprego de tarefas ToM-específicas, mas também outras medidas de processamento cognitivo que pudessem exigir dos examinados o recrutamento de circuitos relacionados à inferência de estados mentais de si mesmo ou de terceiros ou de rastreamento de intencionalidade. Tais medidas incluíram a compreensão de metáforas e ironias, apofenia (a tendência em perceber intencionalidade em movimentos aleatórios), tomada de perspectiva visual e auto-monitoramento. Embora muitos dos estudos selecionados sugerirem que indivíduos com altos escores em instrumentos para avaliação da esquizotipia e/ou familiares de indivíduos esquizofrênicos apresentem déficits no processamento ToM, apontando para um provável caráter traço-dependente da cegueira mental - predispondo ao desenvolvimento de quadros psicóticos, os dados são, ainda, contraditórios. Muitas das respostas às contradições dos achados dizem respeito às metodologias empregadas nos estudos. Conforme afirmado anteriormente, a maior parte dos instrumentos para avaliação de habilidades ToM não foi desenvolvida especificamente para o estudo de populações de indivíduos psicóticos ou vulneráveis à psicose, além de apresentarem limitações intrínsecas, como serem exclusivamente verbais ou não-verbais e não conseguirem, em sua maioria, avaliar o processamento ToM online. Além disso, outras perguntas precisam, ainda, de respostas. Por exemplo, em que magnitude os déficits de processamento cognitivo não associados especificamente à inferência de estados mentais e variáveis relativas à inteligência po- dem estar por trás das baixas pontuações de indivíduos vulneráveis nas tarefas ToM? As respostas a estas questões auxiliarão na compreensão tanto dos mecanismos subjacentes à origem da cegueira mental nestas populações quanto da relação entre déficits da inferência de estados mentais e sintomas psicóticos.

Outro grupo de transtornos psiquiátricos que tem recebido atenção no que se refere à possibilidade de cursarem com cegueira para estados mentais é o dos transtornos do humor. Sabe-se que portadores de transtornos do humor podem desenvolver comprometimento funcional persistente à custa de problemas relacionados ao funcionamento interpessoal. Tal comprometimento predisporia, também, ao aumento do número de episódios de humor (Goldberg, Garno, \& Harrow, 2005). Além disso, pesquisas recentes têm demonstrado que os transtornos do humor cursam com problemas em vários domínios cognitivos, particularmente em funções executivas (Green, Cahill, \& Malghi, 2007). Em relação ao Transtorno Bipolar do Humor (TBH), as pesquisas a respeito do processamento ToM são poucas, quando comparadas ao volume de dados publicados envolvendo portadores de esquizofrenia ou até mesmo indivíduos vulneráveis à psicose. Uma busca feita por nosso grupo na base de dados MEDLINE, por estudos clínicos nos últimos vinte anos, envolvendo portadores de TBH e empregando uma ou mais ferramentas para avaliação de habilidades ToM, resultou em apenas sete artigos (Bora et al., 2005; Inoue, Tonnoka, Yamada, \& Kanba, 2004; Inoue, Yamada, \& Kanba, 2006; Kerr, Dunbar, \& Bentall, 2003; Lahera et al., 2008; Olley et al., 2005; Schenkel, Marlow O'Connor, Moss, Sweeney, \& Pavuluri, 2008). Estes compararam o desempenho de indivíduos bipolares e unipolares eutímicos com controles saudáveis em tarefas ToM (Bora et al., 2005; Inoue et al., 2004; Inoue et al., 2006; Lahera et al., 2008; Olley et al., 2005), bem como a performance nas mesmas tarefas de indivíduos bipolares em fases maníaca, depressiva e em remissão versus controles saudáveis (Kerr et al., 2003) e também o desempenho de crianças e adolescentes portadores de TBH em testes envolvendo mentalização (Schenkel et al., 2008).

As principais conclusões feitas a partir da leitura destes trabalhos são resumidas a seguir: em primeiro lugar, para uma grande parte dos autores, problemas no processamento ToM em bipolares parecem ocorrer tanto nas fases depressivas quanto nas fases maníacas do TBH e na eutimia, sugerindo que elas possam ter um caráter traço-dependente. É importante frisar, contudo, que alguns autores não conseguiram documentar déficits de mentalização em períodos de remissão de episódios maníacos, hipomaníacos ou depressivos do TBH. Em segundo lugar, cabe lembrar que nenhuma amostra avaliada nos trabalhos selecionados examinou indivíduos em episódios mistos, assim como a grande maioria deles não levou em conta a possibilidade de que medicações comumente empregadas no tratamento do transtorno bipolar - como o lítio e os anticonvulsivantes - e comor- 
bidades psiquiátricas comuns no TBH - por exemplo, transtornos de ansiedade e Transtorno de Déficit de Atenção e Hiperatividade - possam ter alguma participação na gênese dos déficits cognitivos sociais observados nos indivíduos avaliados. Por fim, também encontramos limitações metodológicas em muitos dos estudos, como a falta de um controle cognitivo feito por aferição de funcionamento executivo e QI, além de que alguns deles utilizaram ou tarefas ToM puramente verbais ou puramente visuais, o que torna a generalização dos resultados problemática.

\section{Conclusões}

Apesar dos estudos sobre a cegueira mental terem sido inicialmente conduzidos em indivíduos portadores de transtornos do espectro do autismo, eles parecem indicar que déficits ToM podem afetar também indivíduos que sofrem de transtornos psiquiátricos como a esquizofrenia e o TBH. Muitos destes estudos sugerem, ainda, que indivíduos predispostos à esquizofrenia - como os diagnosticados como tendo TEP ou parentes de primeiro grau de esquizofrênicos - e indivíduos bipolares em eutimia possam apresentar algum grau de cegueira mental. Esta explicaria, pelo menos em parte, alguns dos comemorativos clínicos destes quadros psicopatológicos, além de alguns desfechos desfavoráveis dos mesmos, tanto em relação a empobrecimento dos contatos interpessoais, quanto em relação ao aumento do número de episódios de humor ou de psicose. Mais estudos acerca do tema, envolvendo um aprimoramento dos instrumentos utilizados e em seus desenhos experimentais, são necessários para a melhor compreensão do papel da cegueira mental tanto nos transtornos do EA quanto em outras condições psiquiátricas.

\section{Referências}

American Psychiatric Association. (2002). DSM-V-TR: Manual de diagnóstico e estatística de transtornos mentais (4. ed. rev.). Porto Alegre, RS: Artes Médicas.

Baron-Cohen, S. (1995). Mindblindness. An essay on autism and theory of mind. Cambridge, MA: The MIT Press.

Baron-Cohen, S., Leslie, A., \& Frith, U. (1986). Mechanical, behavioural and intentional understanding of picture stories in autistic children. British Journal of Developmental Psychology, 4, 113-125.

Baron-Cohen, S., Wheelwright, S., Hill, J., Raste, Y., \& Plumb, I. (2001). The "Reading the mind in the eyes" test revised version: A study with normal adults, and adults with Asperger syndrome or high-functioning autism. Journal of Child Psychology and Psychiatry, 42(2), 241-251.

Blakemore, S. J., \& Decety, J. (2001). From the perception of action to the understanding of intention. Nature Reviews. Neuroscience, 2(8), 561-567.

Bora, E., Vahip, S., Gonul, A. S., Akdeniz, F., Alkan, M., Ogut, M., et al. (2005). Evidence for theory of mind deficits in euthymic patients with bipolar disorder. Acta Psychiatrica Scandinavica, 112, 110-116.
Bora, E., Yucel, M., \& Pantelis, C. (2009). Theory of mind impairment in schizophrenia: Meta-analysis. Schizophrenia Research, 109(1/3), 1-9.

Brüne, M. (2003). Theory of mind and the role of IQ in chronic disorganized schizophrenia. Schizophrenia Research, 60, 5764.

Brüne, M. (2005). "Theory of mind" in schizophrenia: A review of the literature. Schizophrenia Bulletin, 31(1), 21-42.

Brüne, M., \& Bodenstein, L. (2005). Proverb comprehension reconsidered: 'Theory of mind' and the pragmatic use of language in schizophrenia. Schizophrenia Research, 75(2/3), 233-239.

Brüne, M., \& Brüene-Cohrs, U. (2006). Theory of mind: Evolution, ontogeny, brain mechanisms and psychopathology. Neurosciences and Biobehavioral Reviews, 30, 437-455.

Bullot, N. J., \& Rysiew, P. (2007). A study in the cognition of individual's identity: Solving the problem of singular cognition in object and agent tracking. Consciousness and Cognition, 16, 276-293.

Corcoran, R., Cahil, C., \& Frith, C. D. (1997). The appreciation of visual jokes in people with schizophrenia: A study of 'mentalizing' ability. Schizophrenia Research, 91, 122-131.

Corcoran, R., Mercer, G., \& Frith, C. D. (1995). Schizophrenia, symptomatology and social inference: Investigating "Theory of mind" in people with schizophrenia. Schizophrenia Research, 17, 5-13.

Dennett, D. C. (1987). The intentional stance. Cambridge, MA: MIT Press.

Donald, M. (1993).Origins of the modern mind. Three stages in the evolution of culture and cognition. Cambridge, MA: Harvard University Press.

Dover, C. J., \& Le Couteur, A. (2007). How to diagnose autism. Archives of Disease in Childhood, 92, 540-545.

Dunbar, R. (2002). Grooming, gossip and the evolution of language. Cambridge, MA: Harvard University Press.

Flavell, J. H. (1999). Cognitive development: Children's knowledge about the mind. Annual Reviews of Psychology, 50, 21-45.

Frith, C. D. (1992). The Cognitive Neuropsychiatry of schizophrenia. Hove, UK: Lawrence Erlbaum.

Frith, C. D., \& Frith, U. (1999). Interacting minds: A biological basis. Science, 286, 1692-1695.

Frith, U., \& Happè, F. (1999). Theory of mind and selfconsciousness: What is like to be autistic? Mind \& Language, 14(1), 1-22.

Gazzaniga, M. S., Ivry, R. B., \& Mangun, G. R. (2006). Métodos em Neurociência Cognitiva. Neurociência Cognitiva. A biologia da mente. Porto Alegre, RS: Artmed.

Goldberg, J. F., Garno, J. L., \& Harrow, M. (2005). Long-term remission and recovery in bipolar disorder: A review. Current Psychiatry Reports, 7(6), 456-461.

Gómez, J. C. (2009). Embodying meaning: Insights from primates, autism, and Brentano. Neural Networks, 22, 190-196.

Green, J. M., Cahill, C. M., \& Malhi, G. S. (2007). The cognitive and neurophysiological basis of emotion dysregulation in bipolar disorder. Journal of Affective Disorders, 103, 29-42.

Hardy-Baylé, M. C., Passerieux, C., Claudel, B., Olivier, V., \& Chevalier, J. F. (1994). Communication disorders in schizophrenic patients. Cognitive explanation and clinical reconsideration. Encephale, 20(4), 393-400.

Hogrefe, G. L., Wimmer, H., \& Perner, J. (1986). Ignorance versus false belief: A developmental lag in attribution of epistemic states. Child Development, 57, 567-582. 
Inoue, Y., Tonooka, Y., Yamada, K., \& Kanba, S. (2004). Deficiency of theory of mind in patients with remitted mood disorder. Journal of Affective Disorders, 82, 403-409.

Inoue, Y., Yamada, K., \& Kanba, S. (2006). Deficit in theory of mind is a risk for relapse of major depression. Journal of Affective Disorders, 95, 125-127.

Kerr, N., Dunbar, R. I. M., \& Bentall, R. P. (2003). Theory of mind deficits in bipolar affective disorder. Journal of Affective Disorders, 73, 253-259.

Lahera, G., Montes, J. M., Benito, A., Valdivia, M., Medina, E., Mirapeix, I., et al. (2008). Theory of mind deficit in bipolar disorder: Is it related to a previous history of psychotic symptoms? Psychiatry Research, 161, 309-317.

Langdon, R., \& Coltheart, M. (2004). Recognition of metaphor and irony in young adults: The impact of schizotypal personality traits. Psychiatry Research, 1, 9-20.

Leslie, A. M. (1994). Pretending and believing: Issues in the theory of ToMM. Cognition, 50(1/3), 211-238.

Mc Donald, S., Flanagan, S., Rollins, J., \& Kinch, J. (2003). TASIT: A new clinical tool for assessing social perception after traumatic brain injury. The Journal of Head Trauma Rehabilitation, 18, 219-238.

Olley, A. L., Malhi, G. S., Bachelor, J., Cahill, C. M., Mitchell, P. B., \& Berk, M. (2005). Executive functioning and theory of mind in euthymic bipolar disorder. Bipolar Disorders, 7(Suppl. 5), 43-52.

Perner, J., \& Wimmer, H. (1985). 'John thinks that Mary thinks that...' Attribution of second-order beliefs by $5-10$ years old children. Journal of Experimental Psychology, 39, 437471.

Premack, D., \& Woodruff, G. (1978). Does the chimpanzee have a theory of mind? The Behavioral and Brain Sciences, 1, $515-526$

Rizzolatti, G., \& Sinigaglia, C. (2007). Mirror neurons and motor intentionality. Functional Neurology, 22(4), 205-210.

Rochat, P. (2007). Intentional action arises from early reciprocal exchanges. Acta Psychologica, 124, 8-25.

Schenkel, L. S., Marlow O’Connor, M., Moss, M., Sweeney, J. A., \& Pavuluri, M. N. (2008). Theory of mind and social inference in children and adolescents with bipolar disorder. Psychological Medicine, 38, 791-800.

Sprong, M., Schothorst, P., Vos, E., Hox, J., \& van Engeland, H. (2007). Theory of mind in schizophrenia. Meta-analysis. British Journal of Psychiatry, 191, 5-13.

Stone, V., Baron-Cohen, S., \& Knight, R. (1998). Frontal lobe contributions to theory of mind. Journal of Cognitive Neuroscience, 10, 640-656.

Tonelli, H. A., Alvarez, C., \& da Silva, A. A. (2009). Esquizotipia, alterações no processamento de habilidades "Teoria da mente" e vulnerabilidade à psicose: Uma revisão sistemática. Revista de Psiquiatria Clínica, 36(6), 229-239.

Wimmer, H., \& Perner, J. (1983). Beliefs about beliefs: Representation and constraining function of wrong beliefs in young children's understanding of deception. Cognition, $13,103-128$. 